\title{
Colectomy and desmoid tumours in familial adenomatous polyposis: a systematic review and meta-analysis
}

\author{
Arthur S. Aelvoet ${ }^{1} \cdot$ Daphne Struik $^{1} \cdot$ Barbara A. J. Bastiaansen ${ }^{1} \cdot$ Willem A. Bemelman $^{2} \cdot$ Roel Hompes $^{2}$. \\ Patrick M. M. Bossuyt ${ }^{3} \cdot$ Evelien Dekker $^{1}$ (1)
}

Received: 10 September 2021 / Accepted: 3 January 2022 / Published online: 13 January 2022

(c) The Author(s) 2022

\begin{abstract}
Desmoid tumours (DT) are one of the main causes of death in patients with familial adenomatous polyposis (FAP). Surgical trauma is a risk factor for DT, yet a colectomy is inevitable in FAP to prevent colorectal cancer. This systematic review and meta-analysis aimed to synthesize the available evidence on DT risk related to type, approach and timing of colectomy. A search was performed in MEDLINE, EMBASE and the Cochrane Library. Studies were considered eligible when DT incidence was reported after different types, approaches and timing of colectomy. Twenty studies including 6452 FAP patients were selected, all observational. No significant difference in DT incidence was observed after IRA versus IPAA (OR 0.99, 95\% CI 0.69-1.42) and after open versus laparoscopic colectomy (OR 0.88, 95\% CI 0.42-1.86). Conflicting DT incidences were seen after early versus late colectomy and when analysing open versus laparoscopic colectomy according to colectomy type. Three studies reported a (non-significantly) higher DT incidence after laparoscopic IPAA compared to laparoscopic IRA, with OR varying between 1.77 and 4.09. A significantly higher DT incidence was observed in patients with a history of abdominal surgery (OR 3.40, 95\% CI 1.64-7.03, p=0.001). Current literature does not allow to state firmly whether type, approach, or timing of colectomy affects DT risk in FAP patients. Fewer DT were observed after laparoscopic IRA compared to laparoscopic IPAA, suggesting laparoscopic IRA as the preferred choice if appropriate considering rectal polyp burden. PROSPERO registration number CRD42020161424.
\end{abstract}

Keywords Familial adenomatous polyposis $\cdot$ Colectomy $\cdot$ Desmoid tumours $\cdot$ Meta-analysis

\section{Background}

Prophylactic surgery and intensive endoscopic surveillance has decreased the risk of colorectal cancer (CRC) in patients with familial adenomatous polyposis (FAP) and has improved life expectancy $[1,2]$. Consequently, new

Evelien Dekker

e.dekker@amsterdamumc.nl

1 Department of Gastroenterology and Hepatology, Cancer Center Amsterdam, Amsterdam Gastroenterology Endocrinology Metabolism, Amsterdam UMC, University of Amsterdam, Meibergdreef 9, 1105 AZ Amsterdam, the Netherlands

2 Department of Surgery, Cancer Center Amsterdam, Amsterdam UMC, University of Amsterdam, Amsterdam, the Netherlands

3 Department of Epidemiology and Data Science, Amsterdam UMC, University of Amsterdam, Amsterdam, the Netherlands challenges in the management of FAP arise in this aging population, which are mostly related to extra-colonic manifestations of the disease. Nowadays, one of the most common FAP-related causes of death are desmoid tumours (DT) [3, 4], occurring in $12 \%$ of patients [5]. Desmoid tumours are benign myofibroblastic proliferations, arising most often in the small bowel mesentery or abdominal wall. Intraabdominal DT are a major source of morbidity, as they might cause compression and even perforation of hollow viscera, blood vessels or ureters. Reported risk factors for the development of DT are female sex, a positive family history for DT, a germline mutation in the $A P C$ gene on the 3 ' end of codon 1399, and a history of abdominal surgery [5].

Nearly all patients with FAP undergo a prophylactic colectomy to prevent CRC. Among known risk factors, colectomy might be the only modifiable determinant of DT formation. A different timing, surgical approach, type of colectomy, and reconstruction of continuity may result in differences in DT risk. Up to 85\% of DT develop after 
abdominal surgery [6]. DT also tend to arise shortly after surgery, with a median interval of 3.2 years, highlighting the potential influence of surgical trauma [5]. Most FAP patients undergo total colectomy with ileorectal anastomosis (IRA) or proctocolectomy with ileal pouch-anal anastomosis (IPAA) [7]. The severity of rectal polyp burden is widely used to guide the choice between IRA and IPAA. Some authors advice to perform IRA in patients at high risk of DT, hypothesizing that stretching of the small bowel mesentery and lengthening manoeuvres at the index surgery may trigger DT development when constructing an IPAA $[8,9]$.

The reported cumulative DT incidence after colectomy varies substantially, with proportions ranging from 1.6 to $17.2 \%$ [10-18]. To guide decision-making and to improve the consent process for prophylactic surgery in FAP patients, it would be helpful to review the current evidence for the risk of DT in relation to type, approach, and timing of colectomy. The aim of this study was to systematically review the literature, to calculate summary estimates of the relative risks for DT related to colectomy, and to explore source of heterogeneity in reported results between studies.

\section{Methods}

This systematic review and meta-analysis is summarized in accordance to the Preferred Reporting Items for Systematic Reviews and Meta-analyses (PRISMA) guidelines [19]. The protocol of this review was included in the PROSPERO international register of systematic reviews (CRD42020161424).

\section{Search strategy}

A systematic search was conducted with assistance of a clinical librarian using MEDLINE, EMBASE and the Cochrane Library to identify studies from inception up to 2021. The search (reported in full in Supplementary Material 1) included the following Medical Subject Heading (MeSH) and entry terms: Adenomatous Polyposis Coli (Mesh), APC Genes (Mesh), Adenomatous Polyposis Coli Protein (Mesh), adenomatous polyposis, FAP, familial polyposis, hereditary polyposis, polyposis coli, Aggressive Fibromatosis (Mesh), desmoid, aggressive fibromatosis, fibrous tissue neoplasms, mesenteric fibromatosis. The search was last updated on June 4, 2021. No restrictions were applied on publication date or language. By cross-referencing relevant articles, potential additional studies of interest were identified.

\section{Study selection}

Studies were considered eligible when the corresponding article included estimates of the incidence of DT. Studies were excluded when it was impossible to calculate incidence estimates, for example, when studies only reported on patients with desmoid tumours (DT) and not on the whole study group of FAP patients, or when no comparison between treatment groups was made.

Two reviewers (A.S.A. and D.S.) independently screened all titles and abstracts of identified studies. Subsequently, studies considered potentially eligible were included or excluded based on the corresponding full text report. Disagreements between reviewers were discussed and resolved in consensus meetings.

\section{Data extraction}

Two reviewers (A.S.A. and D.S.) independently extracted data from the reports of included studies using a standardized data extraction form, focussing on the following study characteristics: author, year of publication, country, study design, total number of FAP patients, total number of patients with DT, location of DT, and duration of follow-up. Additionally, the number of patients in each treatment group and number of patients with DT were collected for each comparison, for calculating incidence estimates. Authors were contacted by email to collect additional data from all studies with missing data. Disagreements between reviewers in data extraction were resolved in consensus meetings.

\section{Risk of bias assessment}

The same two reviewers also critically appraised included studies with the Cochrane risk-of-bias tool for non-randomized studies of interventions (ROBINS-I) [20]. This tool evaluates the risk of bias based on seven domains: confounding, selection of participants into the study, classification of interventions, deviations from intended interventions, missing data, measurement of outcomes, and selection of the reported results.

\section{Statistical analysis}

The primary aim was to compare the cumulative incidence of DT after ileorectal anastomosis (IRA) versus proctocolectomy with ileal pouch-anal anastomosis (IPAA). Secondary aims were to make a comparison between open or a laparoscopic colectomy, early versus late colectomy, and between patients with a history of abdominal surgery and those without.

Comparisons of the DT incidence between treatment groups were expressed as odds ratios (OR). Summary estimates of the OR were calculated for comparisons including five or more studies using a random-effects model. OR were considered statistically significant when the $95 \%$ confidence interval did not include 1 . 
Publication bias was assessed by inspection of the constructed funnel plot. Heterogeneity between included studies was evaluated by calculating tau and $I^{2}$ statistics.

All statistical analyses were performed using Review Manager version 5.4 (The Nordic-Cochrane Center, The Cochrane Collaboration, Copenhagen, Denmark).

\section{Results}

A total of 1831 records were identified with the search strategy. After removing duplicates and adding 4 records after cross-referencing, 1111 articles were screened for eligibility based on title and abstract. The full text of 89 articles was subsequently examined, leading to the inclusion of 20 studies (Fig. 1). While 27 studies were initially considered eligible after full-text screening, eight of these studies had to be excluded due to overlapping data within the study period. Six authors were contacted; three of them responded and provided additional data or the full text report of the study.

No randomized controlled trials were found. Eighteen manuscripts reported historical cohort studies and two prospective cohort studies. Study characteristics are summarized in Table 1. Eleven studies had been conducted in Europe, six in the Asia-Pacific and two in America. In total, 6452 patients with FAP were included; one or more DT were reported for 804 patients $(12.5 \%)$. Only three studies mentioned that only symptomatic desmoid tumours were included. All Included studies were observational and international guidelines do not recommend radiologic screening to detect (asymptomatic) desmoid tumours. Therefore, we presume that diagnosed desmoid tumours were mostly symptomatic or detected incidentally at a radiologic examination performed for other reasons. Thirteen studies reported on duration of follow-up, which varied from 44 to 248 months.

\section{Risk of bias}

Results of the risk-of-bias assessment using the ROBINS-I tool are shown in Supplementary Table 1. Fifteen of the twenty studies had a serious or critical risk of confounding bias due to insufficient documentation on previously described risk factors for DT development and insufficient measures to reduce bias. A funnel plot of the relative DT

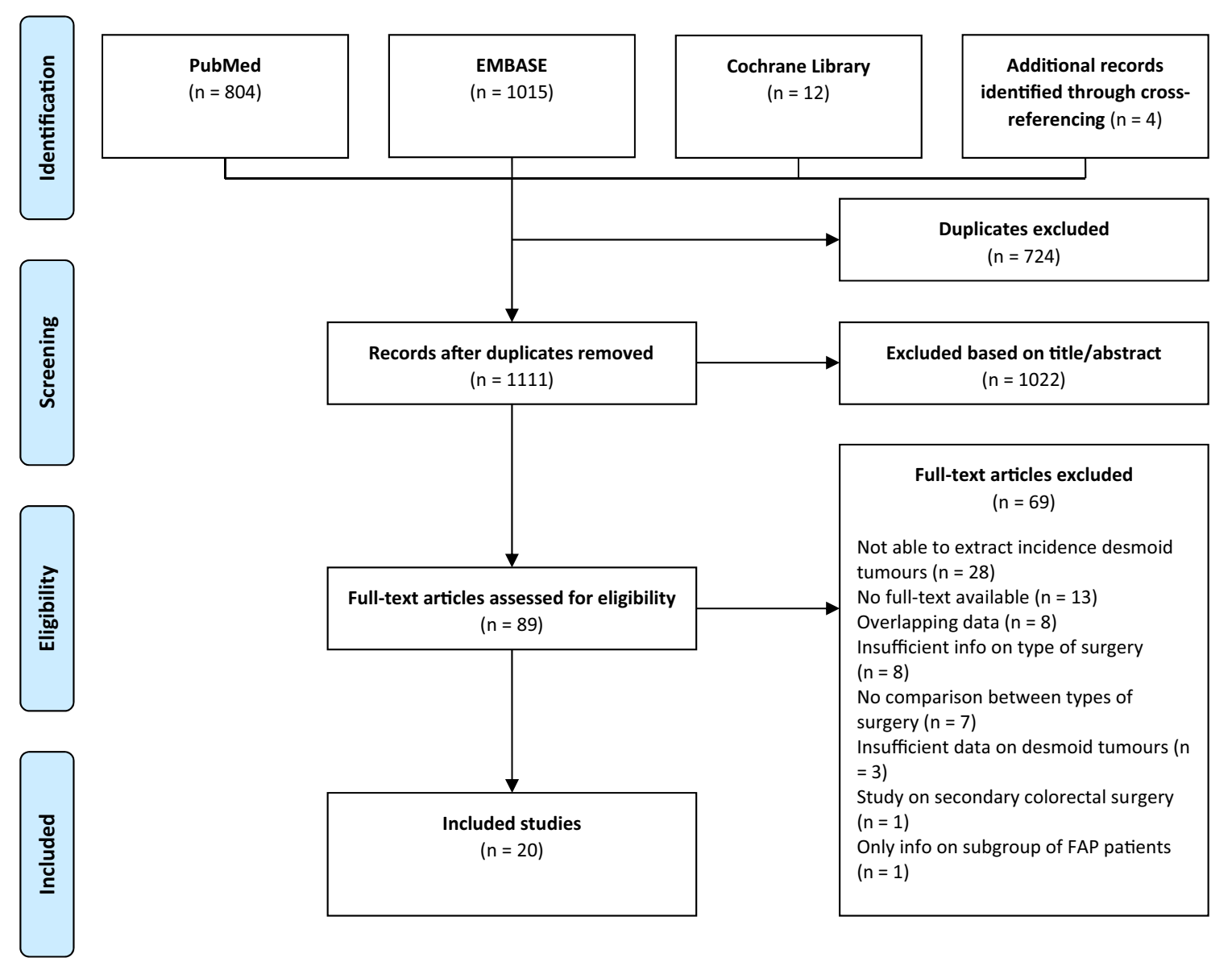

Fig. 1 PRISMA-flowchart 


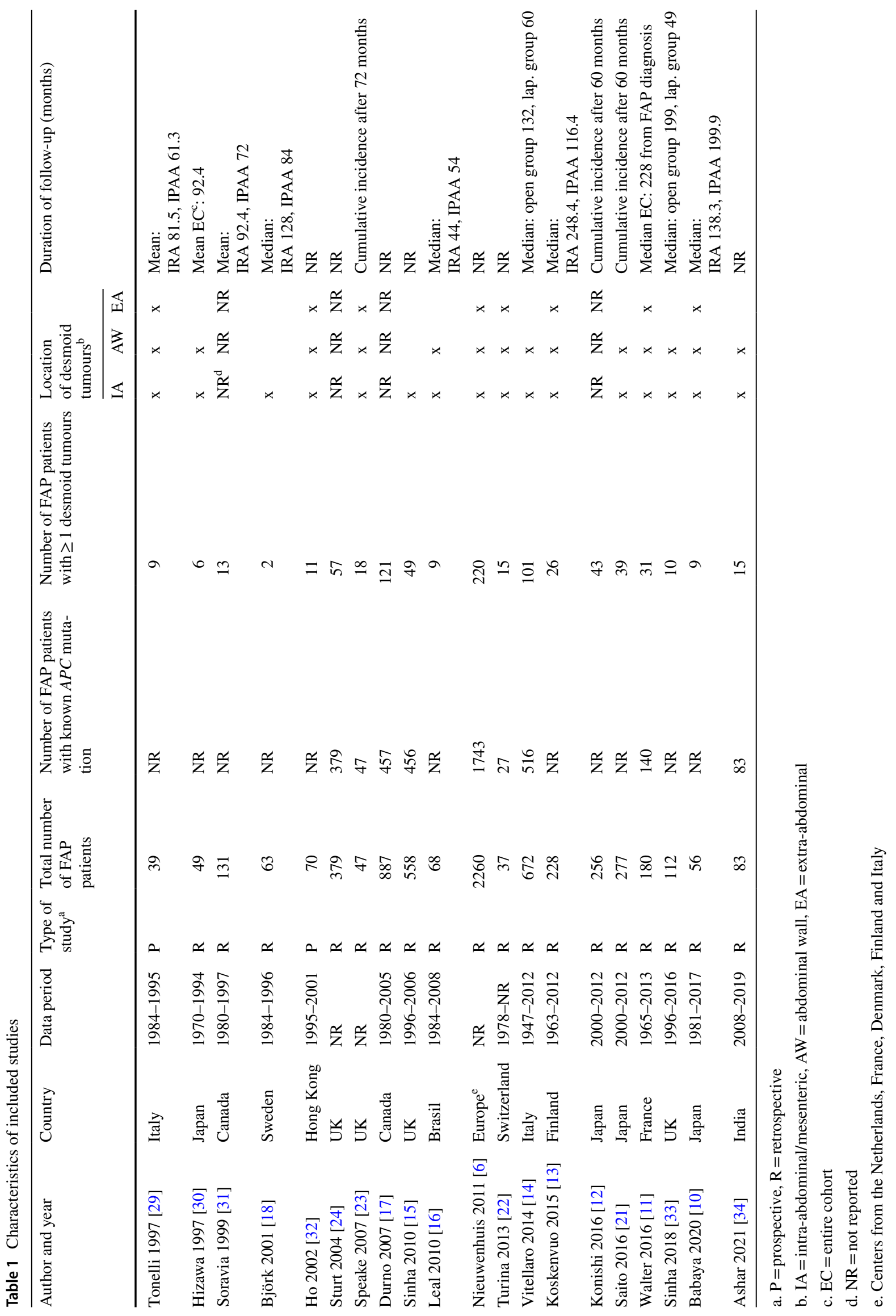


incidence after IRA versus IPAA was constructed to evaluate potential publication bias, showing minor asymmetry, and leading us to conclude on a low risk of publication bias (Fig. 2).

\section{Incidence of desmoid tumours after IRA versus IPAA}

Twelve studies reported on the incidence of DT in FAP patients after they had undergone total colectomy and IRA, comparing these to patients who had undergone proctocolectomy and IPAA (Fig. 3). Within the studies that reported on the age at colectomy, the median or mean age in the IRA group ranged from 26 to 32 , and from 23 to 35 for IPAA. In total, $10.6 \%$ of patients (219 of 2073) developed one or more DT after IRA versus $11.9 \%$ (205 of 1725) after IPAA.

The OR varied from 0.17 to 6.69 between studies. In meta-analysis, a summary OR of 0.99 (95\% CI $0.69-1.42$,

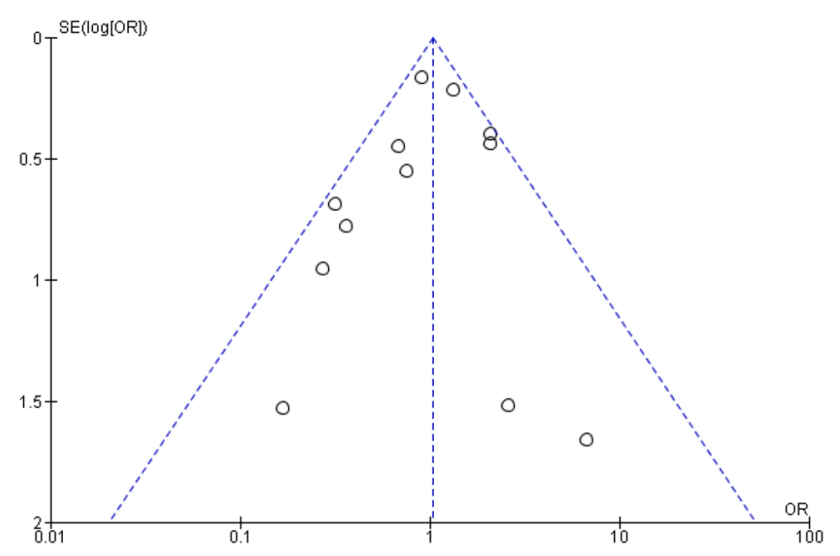

Fig. 2 Funnel plot of studies included in comparison of desmoid tumour incidence after IRA versus IPAA $\mathrm{p}=0.97)$ was calculated with an $\mathrm{I}^{2}$-value of $41 \%$, indicating moderate heterogeneity. Six studies reported on the mean or median duration of follow-up both for patients who underwent IRA and those who underwent IPAA. Studies with a longer duration of follow-up did not show higher DT incidences than studies with a shorter duration of follow-up, as shown in the scatterplot in Fig. 4. Two included studies compared IRA to IPAA in multivariable analysis. Vitellaro et al. [14] reported that undergoing IPAA was a risk factor for DT formation (HR 1.67, 95\% CI 1.06-2.61), adjusted for age at surgery, sex, $A P C$ mutation site, surgical approach and cancer diagnosis. Saito et al. [21] found the same (OR 2.2, 95\% CI 1.1-5.2) when adjusting for age at surgery, sex and surgical purpose (prophylactic/cancer).

\section{Incidence of desmoid tumours after open versus laparoscopic colectomy}

To compare the incidence of DT after open and after laparoscopic surgery, data were extracted from 6 studies (Fig. 5). When ignoring the type of colectomy (IRA or IPAA), the incidence of DT after open and laparoscopic colectomy was 15.2 and $15.5 \%$, respectively. The summary estimate of the OR was 0.88 (95\% CI 0.42-1.86), with substantial heterogeneity between studies ( $\mathrm{I}^{2}$-value of $\left.70 \%\right)$.

Figure 5 shows the forest plots for further comparisons according to the type of colectomy, with widely differing ORs. Odds ratios for DT incidence after open IRA versus open IPAA varied between 0.27 and 4.53 , between 0.41 and 4.74 after open IRA versus laparoscopic IRA, and between 0.07 and 3.08 after open IPAA versus laparoscopic IPAA. Three studies reported a higher DT incidence after laparoscopic IPAA versus laparoscopic IRA, with (non-significant) ORs varying between 1.77 and 4.09.

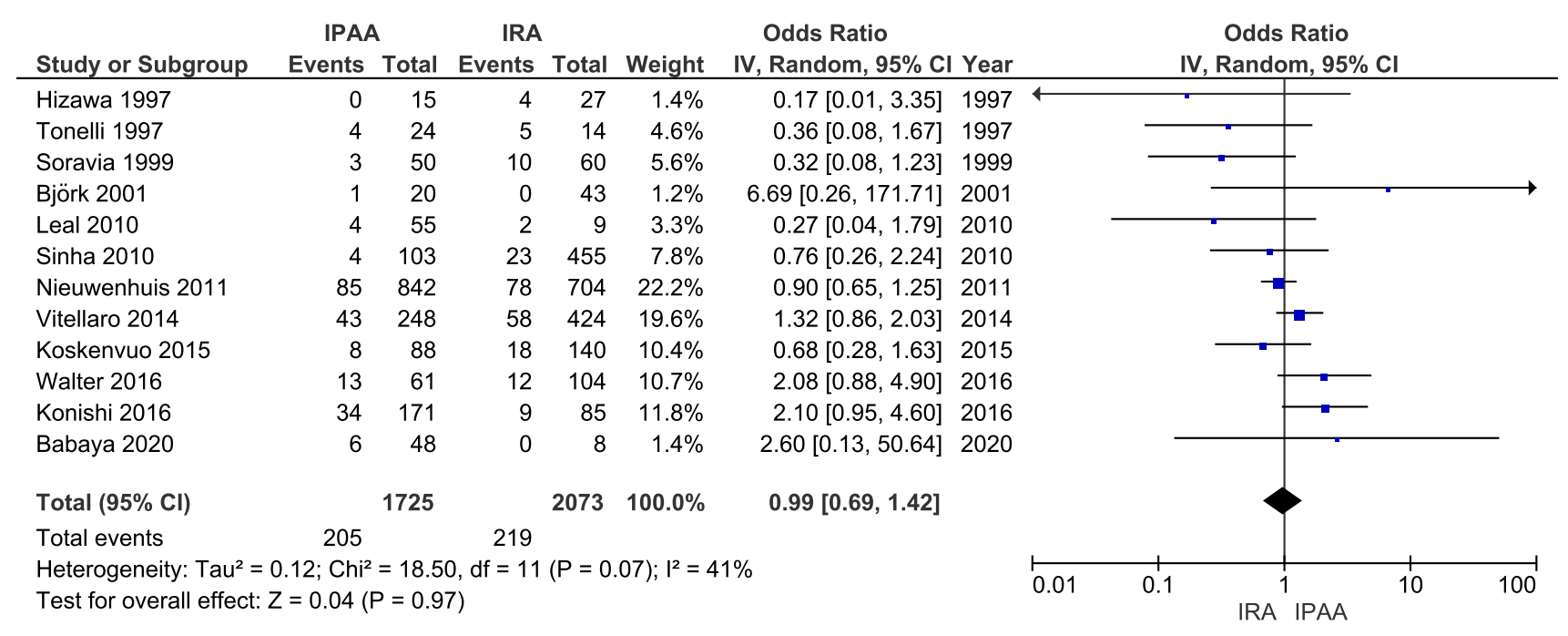

Fig. 3 Incidence of desmoid tumours after IRA versus IPAA 
Fig. 4 Incidence of desmoid tumours versus duration of follow-up. Studies included in scatterplot: 1 . Leal et al. [16] 2. Tonelli et al. [29] 3. Soravia et al. [31] 4. Björk et al. [18] 5 . Koskenvuo et al. [13] 6. Babaya et al. [10]

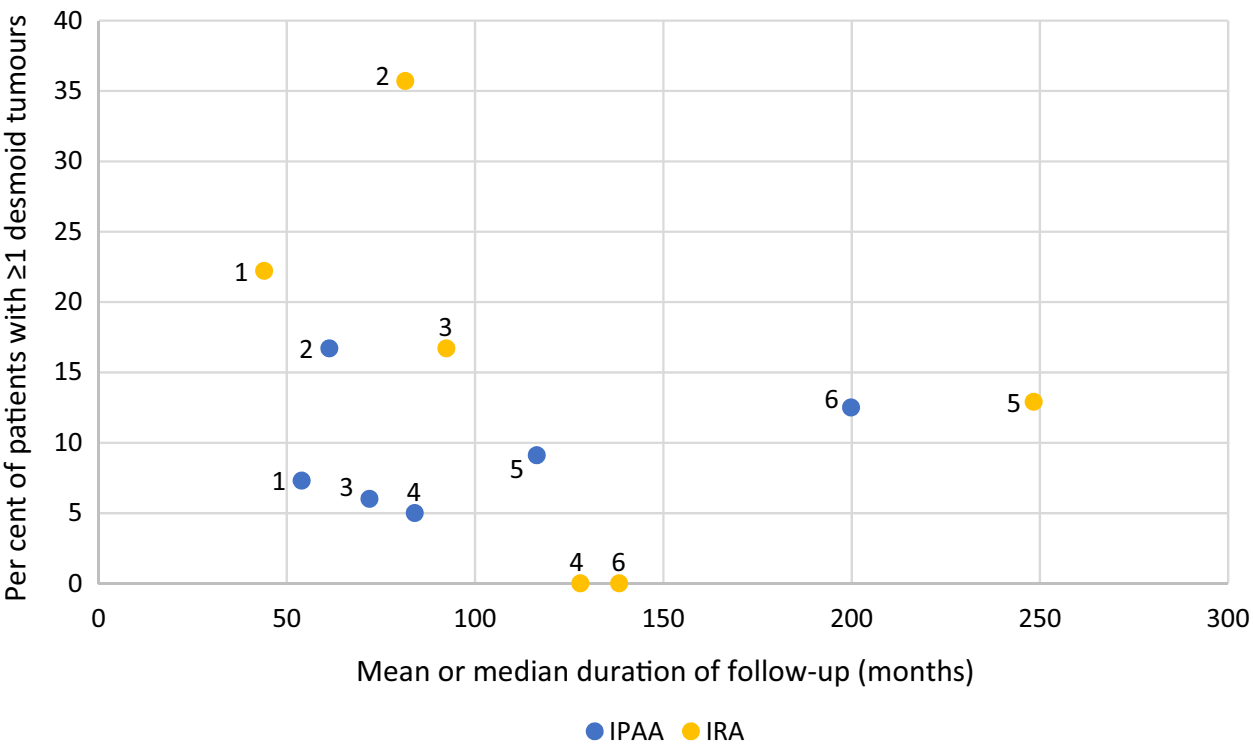

DT developed in $21.4 \%$ of patients after laparoscopic IPAA and in $8.3 \%$ after laparoscopic IRA. Vitellaro et al. [14] found open surgery to be a risk factor for DT formation when adjusting for age at surgery, sex, $A P C$ mutation site, type of surgery and cancer diagnosis (HR 6.84, 95\% CI 1.96-12.98).

\section{Incidence of desmoid tumours after early versus late colectomy}

Four studies reported on DT incidence in FAP patients according to their age at time of colectomy (Fig. 6). Durno et al. [17] and Sinha et al. [15] compared patients who underwent colectomy at the age of 18 or younger and others who underwent surgery after the age of 18 . The ORs in these two studies were 0.69 and 1.32, respectively. Saito et al. [21] and Nieuwenhuis et al. [6] reported on patients that where 30 and 31 respectively or younger compared to those older; ORs for these two studies were 0.63 and 2.79. All studies that performed multivariable analysis did not find timing of colectomy to be a risk factor for DT formation [6, 14, 17, 21]. Only Durno et al. [17] reported that women who had early colectomy (18 years or younger) were more likely to develop DT than women who had colectomy in adulthood (HR 1.77, 95\% CI 1.01-3.09).

\section{Incidence of desmoid tumours in patients with and without a history of abdominal surgery}

Six studies reported on DT incidence in FAP patients with and without a previous history of abdominal surgery (Fig. 7). The cumulative incidence was significantly higher in those who had undergone abdominal surgery previously. The summary estimate of the OR was 3.40 (95\% CI 1.64-7.03, $\mathrm{p}=0.001)$, with an $\mathrm{I}^{2}$-value of $46 \%$ indicating moderate heterogeneity. A history of abdominal surgery was defined as having undergone colectomy with an additional two patients who underwent pancreatic surgery and abdominal lipoma excision in Turina et al. [22] and one patient with history of nephrectomy in Speake et al. [23]. Nieuwenhuis et al. [6] and Sturt et al. [24] did not report on the type of abdominal surgery.

\section{Discussion}

This systematic review summarizes the available evidence on the effects of type, approach, and timing of colectomy in FAP patients on the risk of developing desmoid tumours.

Based on a meta-analysis of available studies, no significant difference in DT risk was observed between patients who underwent total colectomy and IRA and those who underwent proctocolectomy and IPAA. Similarly, no significant difference was observed comparing patients who underwent open versus laparoscopic colectomy. In comparing laparoscopic IRA with laparoscopic IPAA, all three existing studies reported a higher incidence of DT in patients who underwent laparoscopic IPAA, but a similar difference was not observed in those undergoing open procedures.

Although being labelled as a laparoscopic procedure, a laparoscopic IPAA entails partly open surgery including creation of the pouch and lengthening manoeuvres that cannot be safely performed laparoscopically. In addition to trauma related to proctectomy and stretching of the small bowel mesentery, this open segment of the surgery may attribute to 
a. Open colectomy versus laparoscopic colectomy

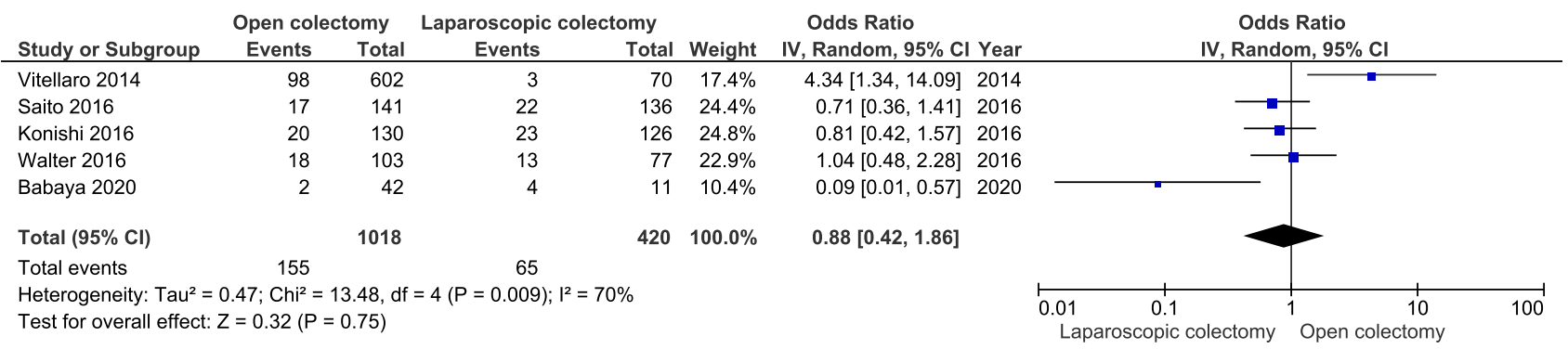

b. Open IPAA versus open IRA

Open IPAA Open IRA Odds Ratio

Study or Subgroup Events Total Events Total IV, Random, 95\% Cl Year

$\begin{array}{llll}4 & 55 & 2 & 9\end{array}$

$\begin{array}{lllllll}\text { Vitellaro } 2014 & 42 & 233 & 56 & 369 & 1.23[0.79,1.91] & 2014\end{array}$

$\begin{array}{lllllll}\text { Konishi } 2016 & 18 & 97 & 2 & 33 & 3.53[0.77,16.13] & 2016\end{array}$

$\begin{array}{lllllll}\text { Babaya } 2020 & 2 & 37 & 0 & 5 & 0.77[0.03,18.38] & 2020\end{array}$

c. Laparoscopic IPAA versus laparoscopic IRA

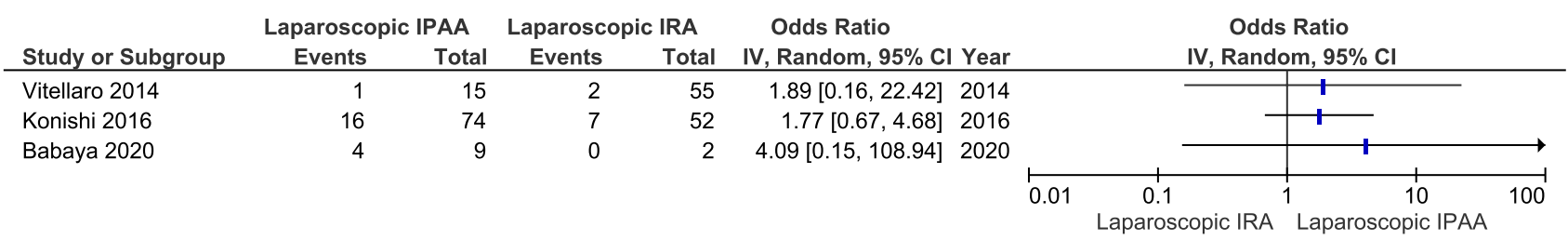

d. Open IRA versus laparoscopic IRA

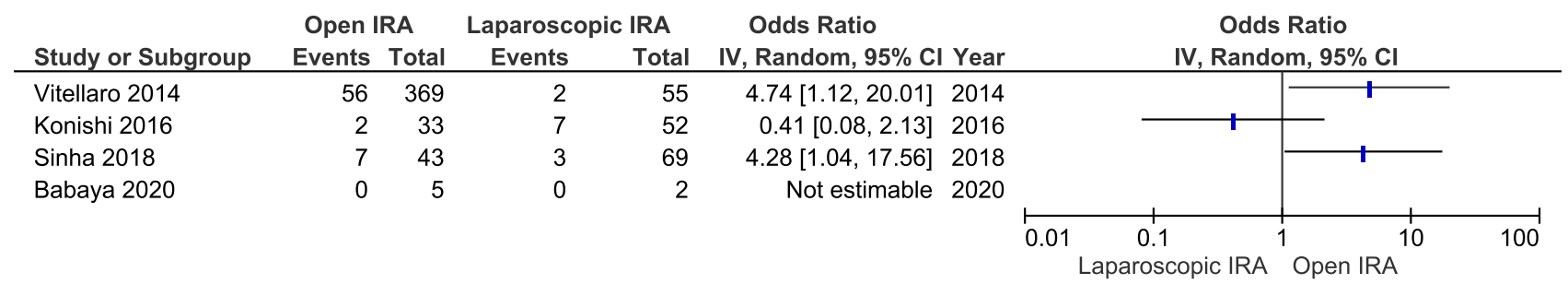

e. Open IPAA versus laparoscopic IPAA

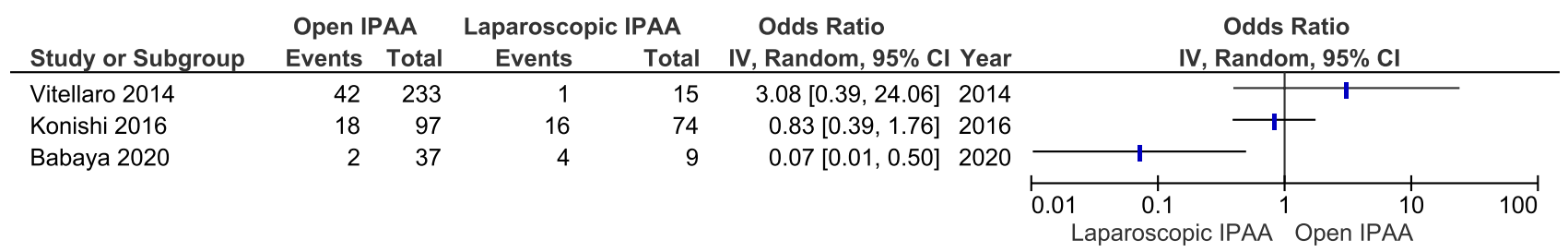

Fig. 5 Incidence of desmoid tumours after open versus laparoscopic colectomy. a. Open colectomy versus laparoscopic colectomy. b. Open IPAA versus open IRA. c. Laparoscopic IPAA versus laparo- scopic IRA. d. Open IRA versus laparoscopic IRA. e. Open IPAA versus laparoscopic IPAA 


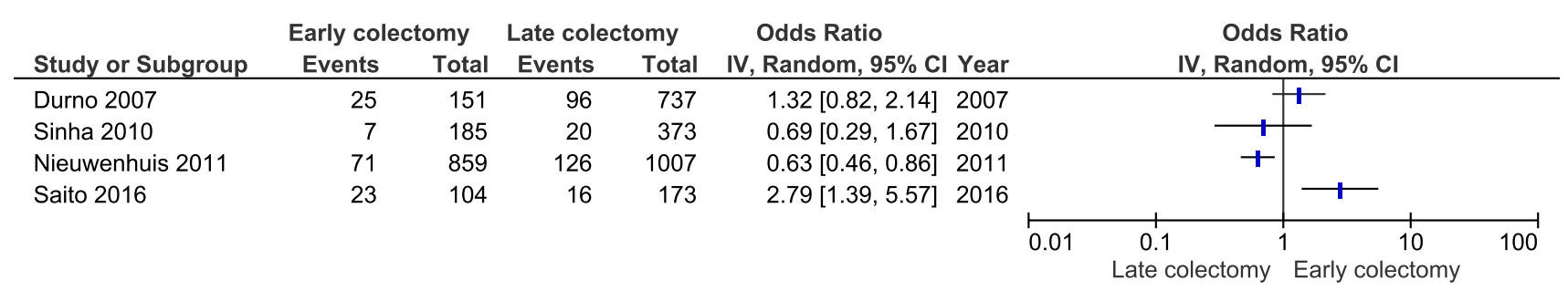

Fig. 6 Early versus late colectomy. Definition early/late colectomy: Durno 2007: early $\leq 18$, late $>18$. Sinha 2010: early $\leq 18$, late $>18$. Nieuwenhuis 2011: early $\leq 31$, late $>31$. Saito 2016: early $\leq 30$, late $>30$

\begin{tabular}{|c|c|c|c|}
\hline \multirow[b]{2}{*}{ Study or Subgroup } & \multicolumn{2}{|c|}{ Prev abdominal surgery } & No prev abdominal surge \\
\hline & Events & Total & Events \\
\hline Ho 2002 & 10 & 52 & 1 \\
\hline Sturt 2004 & 48 & 297 & 2 \\
\hline Speake 2007 & 16 & 22 & 2 \\
\hline Nieuwenhuis 2011 & 199 & 1882 & 21 \\
\hline Turina 2012 & 9 & 18 & 4 \\
\hline Ashar 2021 & 3 & 9 & 12 \\
\hline Total $(95 \% \mathrm{Cl})$ & & 2280 & \\
\hline Total events & 285 & & 42 \\
\hline \multirow{2}{*}{\multicolumn{4}{|c|}{ Heterogeneity: $\mathrm{Tau}^{2}=0.35 ; \mathrm{Chi}^{2}=9.26, \mathrm{df}=5(\mathrm{P}=0.10) ; \mathrm{I}^{2}=46 \%$}} \\
\hline & & & \\
\hline
\end{tabular}

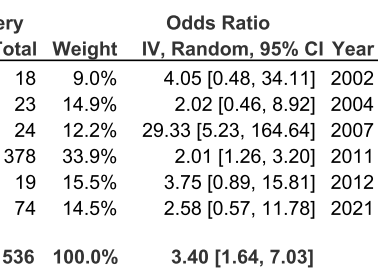

$\begin{array}{lll}536 & 100.0 \% & 3.40\end{array}[1.64,7.03]$

Heterogeneity: $\mathrm{Tau}^{2}=0.35 ; \mathrm{Chi}^{2}=9.26, \mathrm{df}=5(\mathrm{P}=0.10) ; \mathrm{I}^{2}=46 \%$

Test for overall effect: $Z=3.30(P=0.0010)$

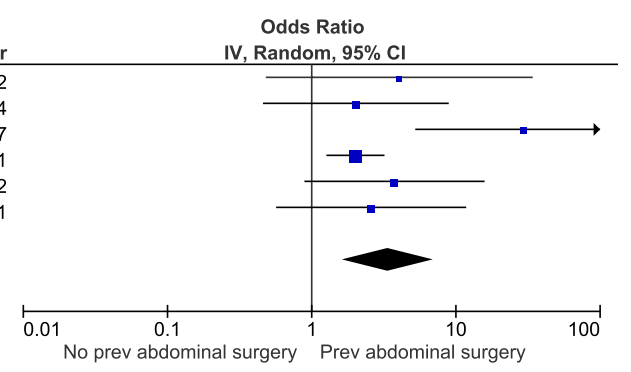

Fig. 7 History of abdominal surgery versus no history of abdominal surgery

DT formation as well. Some of the procedures in the IPAA group might be two-stage procedures with temporary ileostomy. Reversal of the ileostomy results in additional surgical trauma and might thereby contribute to a higher DT risk.

No significant differences were observed between those undergoing colectomy early and those operated later in life. More patients with a history of abdominal surgery developed DT compared to patients without a history of abdominal surgery, as was also observed in a metaanalysis performed by Sinha et al. [5]. However, none of the included studies documented the median age of both groups and patients with a history of abdominal surgery might be older, which potentially contributes to an increased risk of DT. Nevertheless, Nieuwenhuis et al. [6] did not find age to be a risk factor for DT in multivariable analysis.

One more systematic review, by Xie et al. [25], addressed the risk of DT after IRA and IPAA. Similar to the present review, no difference in DT incidence was observed after the different types of colectomy. The review presented here provides additional relevant data and has several strengths. Firstly, 20 studies were included including 6452 FAP patients, of which 804 patients had one or more DT; significantly higher numbers compared to the systematic review performed by Xie et al. (18). More studies were included since multiple comparisons were assessed. For some studies data were initially incomplete but these could be included after authors were contacted. Since the majority of studies on desmoids are performed in a small group of expert centers, the risk of overlapping data was closely assessed and only the most recent study on each outcome from each center was included. Xie et al. [25] included two studies at high risk of overlapping data and some included studies concerned centers that have published on this subject more recently. The present study is the only systematic review investigating the influence of the surgical approach (open or laparoscopic colectomy) on DT risk.

The largest study on DT in FAP patients comprises data from five European registries. It showed that the first diagnosis of DT is made at a young median age of 31 , thus potentially affecting a great part of the life of a patient with FAP [6]. $72 \%$ of DT developed after colorectal surgery, with a median time between surgery and DT diagnosis of 3 years, highlighting the potential influence of surgical trauma in tumour development.

A major difficulty in studying DT lies in the multifactorial etiology. Before drawing conclusions on the influence of types, approaches, and timing of colectomy on DT development, information on other abdominal operations and all known risk factors is needed: sex, DT family history and mutation site on the APC gene [5].

No randomized trials were identified and the available evidence largely stems from historical cohort studies, with limited attempts to correct for confounding. Most of the included studies provided insufficient data on other risk factors for DT development and how these differed between treatment groups. As a result, most studies had serious risk 
of confounding as shown in the risk-of-bias assessment (Table 1).

Patients could be stratified by their risk of DT based on previously reported risk factors before undergoing colectomy. In patients at risk, some authors recommend performing a less extensive IRA $[8,9]$ whereas others advice to perform IPAA [6] arguing that DT formation might prevent a future proctectomy for patients with IRA and advanced rectal polyposis or cancer [26]. This was refuted by Church et al. [27], showing proctectomy was possible in all 26 patients with IRA and DT. Policy differences between centers might introduce selection bias in the included studies in this review, resulting in treatment groups with an overall higher or lower risk of DT at time of colectomy.

Another limitation regards the duration of follow-up, which differed amongst studies and, sometimes, between treatment groups within studies. In six studies, no information on duration of follow-up was available even after contacting the authors. As stated before, DT occur shortly after colorectal surgery in most patients and a longer duration of follow-up might therefore not result in a considerable higher cumulative incidence, as shown in Fig. 4, which included 6 studies with a different duration of follow-up.

Although representing a small part of the total number of DT, patients with extra-abdominal DT were also included in some studies (Table 1). Only DT located in the mesentery or abdominal wall were extracted for construction of forest plots when possible. Studies were not excluded when it was not possible to rule out extra-abdominal desmoids. This is a potential limitation of this review, since formation of these DT are presumably not related to colorectal surgery.

The goal of this systematic review was to assess the risk of DT for guiding surgical decision-making in polyposis patients. Unfortunately, these results do not allow us to state with full confidence that any of the modifiable elements regarding type, approach and timing of colectomy affects the subsequent risk of DT development. A lower incidence after laparoscopic IRA was observed, presumably due to the relatively limited extent of surgical trauma, yet without being able to correct for bias due to confounding and without reaching statistical significance.

Though the initiation of large randomized trials, comparing type and timing of colectomy in patients with FAP over a sufficient duration of follow-up, is desirable, randomization might be challenging, as many other factors also play a role in decision-making. A large multi-center cohort study, with extensive data collection on type and approach of colectomy and all known risk factors, could also add further evidence for deciding whether DT risk should play a role in decision-making for colorectal surgery in FAP.

Since DT most often develop after colorectal surgery in FAP patients, patients at high risk of DT formation based on known risk factors may benefit from postponing colectomy, if feasible considering the severity of the colonic polyposis. As shown in this review, this will presumably not lead to an overall lower risk of DT but could result in DT formation at an older age. DT are less often diagnosed in older patients [5] and a peak incidence is observed in patients in their 20's-30's. This peak might be caused by the fact most patients undergo colectomy in this time of their life more than their age itself. Pregnancy in this period might also elicit DT formation in women [28], although Nieuwenhuis et al. [26] did not find pregnancy to be a risk factor for DT development.

When a clear indication for colectomy is set, robust evidence-based recommendations on the preferred type and approach of colectomy to reduce post-operative desmoid risk cannot be given. Based on current literature, decision-making on type and timing of colectomy should primarily be guided by rectal polyp burden [7,9] . This should always be a shared-decision process with the patient, respecting social factors and potential pregnancy wish in women. In this era of minimal invasive surgery a laparoscopic IRA, when feasible in terms of rectal polyp burden, might be the procedure of choice, possibly resulting in the lowest risk of desmoid formation.

Supplementary Information The online version contains supplementary material available at https://doi.org/10.1007/s10689-022-00288-y.

Acknowledgements The authors thank F.S. van Etten - Jamaludin, clinical librarian, for assistance in performing the literature search used for this review.

Author contributions ASA, PMMB, ED: Concept and design. ASA, DS, BAJB, WAB, RH, PMMB, ED: Analysis and interpretation of the data. ASA: Drafting of the article including figures. ASA, DS, BAJB, WAB, RH, PMMB, ED: Critical revision of the article for important intellectual content. ASA, DS, BAJB, WAB, RH, PMMB, ED: Final approval of the article.

Funding No funding was received for conducting this study.

Data availability The data that support the findings of this study are available from the corresponding author (E.D.) upon request.

Code availability Not applicable.

\section{Declarations}

Conflict of interest Arthur S. Aelvoet: none to declare. Daphne Struik: none to declare. Barbara A.J. Bastiaansen: speakers' fee from Olympus, Tillotts Pharma AG and Ovesco Endoscopy AG. Willem A. Bemelman: VIFOR research grants, Takeda, Braun and Johnson consulting and speakers fee. Roel Hompes: none to declare. Patrick M.M. Bossuyt: none to declare. Evelien Dekker: endoscopic equipment on loan of FujiFilm and Olympus, receive a research grant from FujiFilm. I have received honorarium for consultancy from FujiFilm, Olympus, Tillots, GI Supply, CPP-FAP, PAION and Ambu, and speakers' fees from Olympus, Roche, GI Supply, Norgine and FujiFilm. 
Ethical approval Not applicable.

Consent to participate Not applicable.

Consent for publication Not applicable.

Open Access This article is licensed under a Creative Commons Attribution 4.0 International License, which permits use, sharing, adaptation, distribution and reproduction in any medium or format, as long as you give appropriate credit to the original author(s) and the source, provide a link to the Creative Commons licence, and indicate if changes were made. The images or other third party material in this article are included in the article's Creative Commons licence, unless indicated otherwise in a credit line to the material. If material is not included in the article's Creative Commons licence and your intended use is not permitted by statutory regulation or exceeds the permitted use, you will need to obtain permission directly from the copyright holder. To view a copy of this licence, visit http://creativecommons.org/licenses/by/4.0/.

\section{References}

1. Mallinson EK, Newton KF, Bowen J, Lalloo F, Clancy T, Hill J et al (2010) The impact of screening and genetic registration on mortality and colorectal cancer incidence in familial adenomatous polyposis. Gut 59(10):1378-1382

2. Karstensen JG, Burisch J, Pommergaard HC, Aalling L, Højen $\mathrm{H}$, Jespersen $\mathrm{N}$ et al (2019) Colorectal cancer in individuals with familial adenomatous polyposis, based on analysis of the Danish polyposis registry. Clin Gastroenterol Hepatol 17(11):2294-300. e1

3. Ghorbanoghli Z, Bastiaansen BA, Langers AM, Nagengast FM, Poley JW, Hardwick JC et al (2018) Extracolonic cancer risk in Dutch patients with APC (adenomatous polyposis coli)-associated polyposis. J Med Genet 55(1):11-14

4. de Campos FG, Perez RO, Imperiale AR, Seid VE, Nahas SC, Cecconello I (2010) Evaluating causes of death in familial adenomatous polyposis. J Gastrointest Surg 14(12):1943-1949

5. Sinha A, Tekkis PP, Gibbons DC, Phillips RK, Clark SK (2011) Risk factors predicting desmoid occurrence in patients with familial adenomatous polyposis: a meta-analysis. Colorectal Dis 13(11):1222-1229

6. Nieuwenhuis MH, Lefevre JH, Bülow S, Järvinen H, Bertario L, Kernéis S et al (2011) Family history, surgery, and APC mutation are risk factors for desmoid tumors in familial adenomatous polyposis: an international cohort study. Dis Colon Rectum 54(10):1229-1234

7. Tudyka VN, Clark SK (2012) Surgical treatment in familial adenomatous polyposis. Ann Gastroenterol 25(3):201-206

8. Church J (2017) Deciding on an IRA vs. IPAA for FAP. pp 337-46

9. Chittleborough TJ, Warrier SK, Heriot AG, Kalady M, Church J (2017) Dispelling misconceptions in the management of familial adenomatous polyposis. ANZ J Surg 87(6):441-445

10. Babaya A, Yamano T, Matsubara T, Takenaka Y, Song J, Kimura $\mathrm{K}$ et al (2020) Long-term clinical outcomes and follow-up status in Japanese patients with familial adenomatous polyposis after radical surgery: a descriptive, retrospective cohort study from a single institute. Int J Colorectal Dis 35(4):675-684

11. Walter T, Zhenzhen Wang C, Guillaud O, Cotte E, Pasquer A, Vinet $O$ et al (2017) Management of desmoid tumours: a large national database of familial adenomatous patients shows a link to colectomy modalities and low efficacy of medical treatments. United Eur Gastroenterol J 5(5):735-741
12. Konishi $\mathrm{T}$, Ishida $\mathrm{H}$, Ueno $\mathrm{H}$, Kobayashi $\mathrm{H}$, Hinoi $\mathrm{T}$, Inoue $\mathrm{Y}$ et al (2016) Feasibility of laparoscopic total proctocolectomy with ileal pouch-anal anastomosis and total colectomy with ileorectal anastomosis for familial adenomatous polyposis: results of a nationwide multicenter study. Int J Clin Oncol 21(5):953-961

13. Koskenvuo L, Mustonen H, Renkonen-Sinisalo L, Järvinen HJ, Lepistö A (2015) Comparison of proctocolectomy and ileal pouch-anal anastomosis to colectomy and ileorectal anastomosis in familial adenomatous polyposis. Fam Cancer 14(2):221-227

14. Vitellaro M, Sala P, Signoroni S, Radice P, Fortuzzi S, Civelli EM et al (2014) Risk of desmoid tumours after open and laparoscopic colectomy in patients with familial adenomatous polyposis. Br J Surg 101(5):558-565

15. Sinha A, Tekkis PP, Neale KF, Phillips RK, Clark SK (2010) Risk factors predicting intra-abdominal desmoids in familial adenomatous polyposis: a single centre experience. Tech Coloproctol 14(2):141-146

16. Leal RF, Silva PV, Ayrizono Mde L, Fagundes JJ, Amstalden EM, Coy CS (2010) Desmoid tumor in patients with familial adenomatous polyposis. Arq Gastroenterol 47(4):373-378

17. Durno C, Monga N, Bapat B, Berk T, Cohen Z, Gallinger S (2007) Does early colectomy increase desmoid risk in familial adenomatous polyposis? Clin Gastroenterol Hepatol 5(10):1190-1194

18. Björk J, Akerbrant H, Iselius L, Svenberg T, Oresland T, Påhlman L et al (2001) Outcome of primary and secondary ileal pouch-anal anastomosis and ileorectal anastomosis in patients with familial adenomatous polyposis. Dis Colon Rectum 44(7):984-992

19. Liberati A, Altman DG, Tetzlaff J, Mulrow C, Gøtzsche PC, Ioannidis JP et al (2009) The PRISMA statement for reporting systematic reviews and meta-analyses of studies that evaluate health care interventions: explanation and elaboration. J Clin Epidemiol 62(10): e1-34

20. Sterne JA, Hernán MA, Reeves BC, Savović J, Berkman ND, Viswanathan $\mathrm{M}$ et al (2016) ROBINS-I: a tool for assessing risk of bias in non-randomised studies of interventions. BMJ 355:4919

21. Saito $Y$, Hinoi T, Ueno H, Kobayashi H, Konishi T, Ishida $F$ et al (2016) Risk factors for the development of desmoid tumor after colectomy in patients with familial adenomatous polyposis: multicenter retrospective cohort study in Japan. Ann Surg Oncol 23(Suppl 4):559-565

22. Turina M, Pavlik CM, Heinimann K, Behrensmeier F, Simmen HP (2013) Recurrent desmoids determine outcome in patients with Gardner syndrome: a cohort study of three generations of an APC mutation-positive family across 30 years. Int J Colorectal Dis 28(6):865-872

23. Speake D, Evans DG, Lalloo F, Scott NA, Hill J (2007) Desmoid tumours in patients with familial adenomatous polyposis and desmoid region adenomatous polyposis coli mutations. Br J Surg 94(8):1009-1013

24. Sturt NJ, Gallagher MC, Bassett P, Philp CR, Neale KF, Tomlinson IP et al (2004) Evidence for genetic predisposition to desmoid tumours in familial adenomatous polyposis independent of the germline APC mutation. Gut 53(12):1832-1836

25. Xie M, Chen Y, Wei W, He X, Li X, Lian L et al (2020) Does ileoanal pouch surgery increase the risk of desmoid in patients with familial adenomatous polyposis? Int J Colorectal Dis 35(8):1599-1605

26. Nieuwenhuis MH, De Vos Tot Nederveen Cappel W, Botma A, Nagengast FM, Kleibeuker JH, Mathus-Vliegen EM et al (2008) Desmoid tumors in a dutch cohort of patients with familial adenomatous polyposis. Clin Gastroenterol Hepatol 6(2):215-219

27. Church JM, Xhaja X, Warrier SK, Laguardia L, O'Malley M, Burke $\mathrm{C}$ et al (2014) Desmoid tumors do not prevent proctectomy following abdominal colectomy and ileorectal anastomosis in patients with familial adenomatous polyposis. Dis Colon Rectum 57(3):343-347 
28. Clark SK, Neale KF, Landgrebe JC, Phillips RK (1999) Desmoid tumours complicating familial adenomatous polyposis. Br J Surg 86(9):1185-1189

29. Tonelli F, Valanzano R, Monaci I, Mazzoni P, Anastasi A, Ficari F (1997) Restorative proctocolectomy or rectum-preserving surgery in patients with familial adenomatous polyposis: results of a prospective study. World J Surg 21(6):653-658 (discussion 9)

30. Hizawa K, Iida M, Mibu R, Aoyagi K, Yao T, Fujishima M (1997) Desmoid tumors in familial adenomatous polyposis/Gardner's syndrome. J Clin Gastroenterol 25(1):334-337

31. Soravia C, Klein L, Berk T, O’Connor BI, Cohen Z, McLeod RS (1999) Comparison of ileal pouch-anal anastomosis and ileorectal anastomosis in patients with familial adenomatous polyposis. Dis Colon Rectum 42(8):1028-1033 (discussion 33-34)

32. Ho JW, Chu KM, Tse CW, Yuen ST (2002) Phenotype and management of patients with familial adenomatous polyposis in Hong
Kong: perspective of the Hereditary Gastrointestinal Cancer Registry. Hong Kong Med J 8(5):342-347

33. Sinha A, Burns EM, Latchford A, Clark SK (2018) Risk of desmoid formation after laparoscopic versus open colectomy and ileorectal anastomosis for familial adenomatous polyposis. BJS Open 2(6):452-455

34. Ashar S, Lipsa A, Khan N, Sarin R (2021) High cumulative risk of colorectal cancers and desmoid tumours and fibromatosis in South Asian APC mutation carriers. J Med Genet. https://doi.org/ 10.1136/jmedgenet-2021-107731

Publisher's Note Springer Nature remains neutral with regard to jurisdictional claims in published maps and institutional affiliations. 